\section{O que os cartões de pré-natal das gestantes revelam sobre a assistência nos serviços do SUS da Região Metropolitana da Grande Vitória, Espírito Santo, Brasil?}

\author{
Prenatal patient cards and quality of prenatal care \\ in public health services in Greater Metropolitan \\ Vitória, Espírito Santo State, Brazil
}

\author{
${ }^{1}$ Departamento de Medicina \\ Social, Universidade Federal \\ do Espírito Santo, Vitória, \\ Brasil. \\ 2 Escola Nacional de Saúde \\ Pública Sergio Arouca, \\ Fundação Oswaldo Cruz, Rio \\ de Janeiro, Brasil. \\ 3 Programa de Pós-graduação \\ em Saúde Coletiva, \\ Universidade Federal do \\ Espírito Santo, Vitória, Brasil. \\ Correspondência \\ E. T. Santos Neto \\ Departamento de Medicina \\ Social, Universidade Federal \\ do Espírito Santo. \\ Av. Marechal Campos 1468, \\ Vitória, ES \\ 29040-090, Brasil. \\ edsontheodoro@uol.com.br
}

\begin{abstract}
This study aimed to assess the completeness of prenatal care information on the patients' prenatal care cards, according to coverage by various public health services: Family Health Strategy (FHS), Community-Based Health Workers' Program (CBHWP), and traditional Primary Care Units (PCU) in Greater Metropolitan Vitória, Espírito Santo State, Brazil. In a cross-sectional study, 1,006 prenatal cards were randomly selected from postpartum women at maternity hospitals in the metropolitan area. Completeness of the cards was assessed according to the criteria proposed by Romero \& Cunha, which measure the quality on a scale from excellent $(<$ $5 \%$ incomplete cards) to very bad (>50\% incomplete cards). In general, completion of information on the cards was bad (> 20\% incomplete), but cards were filled out better in the FHS than in the CBHWP and PCU, especially for tetanus vaccination ( $p=0.016)$ and gestational weight ( $p=$ 0.039 ). In conclusion, the quality of prenatal care in the public health system in Greater Metropolitan Vitória fails to meet the Brazilian national guidelines for maternal and child health.
\end{abstract}

Maternal and Child Health; Prenatal Care; Health Evaluation; Quality of Health Care; Health Public Policy
Edson Theodoro dos Santos Neto 1,2 Adauto Emmerich Oliveira 1 Eliana Zandonade 3 Silvana Granado Nogueira da Gama ${ }^{2}$ Maria do Carmo Leal 2

\section{Introdução}

O Cartão da Gestante foi criado no Brasil em 1988, com o propósito de armazenar informações, facilitando a comunicação entre os profissionais que realizavam a assistência pré-natal e os que realizavam o parto nas maternidades 1 . Seu uso se popularizou nos serviços de saúde pública, funcionando como um mecanismo de comunicação entre os níveis de atenção ${ }^{2}$. Por isso, recomenda-se que nele deva conter o máximo de informações 3 , evitando que, no momento do parto, procedimentos ou exames que protejam o feto e a mãe sejam negligenciados ou repetidos desnecessariamente 4 .

Além do seu papel no referenciamento ao parto, os cartões de gestantes funcionam como fonte de informação para o Sistema de Informações sobre Nascidos Vivos (SINASC) 5 e são utilizados em estudos que avaliam a qualidade da assistência pré-natal 6 para fundamentar políticas de saúde materno-infantil no Sistema Único de Saúde (SUS). Sua preservação pela parturiente vem sendo muito valorizada, visto que quase todas chegam para o parto com seus respectivos cartões 7,8. Contudo, o cartão está sujeito à incompletude de registros pelos profissionais que assistem ao pré-natal e às perdas ou extravios pela gestante que, algumas vezes, têm dificuldades quanto ao entendimento dos registros anotados neles 9 . 
A ausência de informações sobre a saúde materno-infantil nos cartões pode expressar diretamente a qualidade dos serviços de assistência pré-natal e limitar a produção de dados para gerar informações fundamentais à organização e ao planejamento dos serviços pré-natais 10 . Nesse sentido, devido à insuficiência histórica de informações relevantes para elaboração de políticas no campo da saúde materno-infantil, a partir de 1991 o Programa Agentes Comunitários de Saúde (PACS) contribuiu substancialmente para produção de informações que possibilitaram diagnósticos situacionais em saúde em vários locais do Brasil 11. Aos municípios habilitados que não alimentavam permanentemente os sistemas de informação, como o SINASC, suspendia-se o cadastramento e o repasse de recursos federais ao programa 12 .

O PACS pressupõe que agentes comunitários de saúde (ACS) e enfermeiros numa área adstrita, dentre outras ações de promoção da saúde e prevenção de agravos, identifiquem e encaminhem gestantes ao pré-natal, acompanhem a vacinação periódica por meio do Cartão da Gestante, identifiquem as situações de risco e as encaminhem aos setores responsáveis 12 .

No intuito de ampliar o escopo do PACS, o Programa Saúde da Família (PSF) reorientou o modelo assistencial do sistema público de saúde brasileiro, facilitando o levantamento de informações de saúde pelo incentivo à cobertura universal, num território definido, sob a égide do princípio da vigilância da saúde. Dentre outras atividades, a incorporação do médico generalista na equipe de saúde permitiu executar as ações de assistência em todos os ciclos da vida 13. No âmbito da assistência pré-natal, verificam-se vantagens da implantação do PSF sobre as unidades básicas de saúde (UBS) tradicionais 14. Nesse sentido, espera-se que no PSF seja realizado o máximo de atividades educativas, clínicas e diagnósticas, qualificando a assistência pré-natal.

Embora apresente vantagens em potencial, o Estratégia Saúde da Família (ESF) ainda não alcança todos os municípios do Brasil. Em alguns deles convivem as UBS tradicionais, o PACS e ESF, seguindo lógicas distintas dentro de um mesmo território. Por isso, este estudo se propõe a avaliar a completude de informações sobre assistência pré-natal nos cartões de gestantes, segundo o tipo de serviços de saúde pública, na Região Metropolitana da Grande Vitória, Espírito Santo, Brasil.

\section{Métodos}

Um estudo epidemiológico seccional foi conduzido na Região Metropolitana da Grande Vitória, que é formada pelos municípios de Cariacica, Fundão, Guarapari, Serra, Viana, Vila Velha e Vitória. Esses sete municípios abrigam quase a metade da população total do Espírito Santo (46\%) e $57 \%$ da população urbana do estado. Foram responsáveis por $65 \%$ do Produto Interno Bruto (PIB) de todo estado em 2008, e juntos somam uma área territorial de $2.331,01 \mathrm{~km}^{2}$ (Instituto de Apoio à Pesquisa e ao Desenvolvimento Jones dos Santos Neves. http://www.ijsn.es.gov.br/, acessado em 09/Jul/2010).

Em relação aos indicadores de mortalidade infantil e materna, no ano de 2007, Fundão apresentou o maior coeficiente de mortalidade infantil (16,5/1.000) e Vitória e Guarapari registraram o menor coeficiente (11,5/1.000) (Departamento de Informática do SUS. http://tabnet.datasus. gov.br/cgi/deftohtm.exe?sim/cnv/inf10es.def, acessado em 02/Set/2008). No mesmo ano, os municípios de Viana, Fundão e Guarapari não apresentaram nenhuma morte materna. Em oposição, Cariacica registrou a razão de mortalidade materna igual a 122,3 mortes para cada grupo de 100.000 nascidos vivos, enquanto em Vitória este indicador foi de 45,1/100.000 (Departamento de Informática do SUS. http://tabnet. datasus.gov.br/cgi/deftohtm.exe?sim/cnv/ma t10es.def, acessado em 02/Set/2008).

No que se refere aos indicadores relativos à rede de serviços de saúde na atenção básica da Região Metropolitana da Grande Vitória, os dados de 2006 do Sistema de Informação da Atenção Básica (SIAB) mostram que a cobertura do ESF da população adstrita chegou a quase $100 \%$ no Município de Fundão, entretanto, não chega a $21 \%$ nos municípios de Cariacica, Guarapari e Serra (Departamento de Informática do SUS. http: / / www2.datasus.gov.br/SIAB/index.php, acessado em 02/Set/2008). Além disso, nos sete municípios dessa região, situam-se oito maternidades públicas e conveniadas ao SUS, que assistem às puérperas produzindo cerca de $80 \%$ dos partos.

O universo amostral foi composto por todas as mulheres que se internaram em maternidades públicas ou conveniadas ao SUS localizadas na Região Metropolitana da Grande Vitória, por ocasião do parto, no período de abril a setembro de 2010. O tamanho da amostra foi definido considerando a população de 17.980 nascidos vivos em 2007, uma proporção esperada igual a 50\%, correspondente à falta de informação prévia sobre este percentual, uma precisão desejada de $4 \%$, o efeito do desenho igual a 1,5 e o 
nível de significância de 5\%, resultando em 872 mulheres. Aumentou-se em cerca de $30 \%$ para considerar as possíveis perdas, recusas ou ausência do Cartão da Gestante no local do parto, o que resultou em 1.131 abordagens e 1.006 cartões avaliados. A representatividade da amostra foi garantida obedecendo à estratificação de acordo com as seguintes proporções: Cariacica (22,6\%), Fundão (1\%), Guarapari (6,3\%), Serra $(26,3 \%)$, Viana $(3,7 \%)$, Vila Velha $(22,2 \%)$ e Vitória $(17,9 \%)$.

Sete entrevistadoras de campo foram selecionadas após a aprovação em testes teóricos e práticos do curso de treinamento de entrevistadores, promovido por professores da Universidade Federal do Espírito Santo (UFES) e da Escola Nacional de Saúde Pública Sergio Arouca, Fundação Oswaldo Cruz (ENSP/Fiocruz). Além disso, um estudo piloto foi conduzido com 67 puérperas - não incluídas no estudo principal para aprimoramento do formulário de pesquisa e treinamento das entrevistadoras.

Essas entrevistadoras visitavam no mínimo uma vez por semana todas as oito maternidades incluídas no estudo, abordavam mulheres no momento após o parto. No dia da visita, todas as puérperas residentes em um dos municípios da Região Metropolitana da Grande Vitória eram listadas em ordem aleatória, em seguida, realizavase a amostragem sistemática intercalando-se de duas em duas. Na abordagem, excluía-se as mulheres que não tinham cartão, as que realizaram acompanhamento pré-natal (todo ou parte) no sistema privado e as que foram acompanhadas em municípios fora da Região Metropolitana da Grande Vitória.

Após a identificação das puérperas sorteadas, as entrevistadoras explicavam os objetivos da pesquisa e solicitavam a assinatura do Termo de Consentimento Livre e Esclarecido, segundo as recomendações aprovadas pelo Comitê de Ética em Pesquisa do Centro de Ciências da Saúde da UFES, em 4 de novembro de 2009, sob protocolo no. 93/2009. Em seguida o Cartão da Gestante era solicitado e copiado na íntegra. No formulário de pesquisa, as entrevistadoras anotavam o endereço completo das puérperas e os locais de realização do acompanhamento prénatal. As variáveis do estudo foram construídas com base nas informações contidas nos cartões e nos formulários.

Cada registro nos campos do cartão foi avaliado segundo temáticas: variáveis utilizadas no cadastramento e no registro diário do Programa de Humanização do Pré-natal e Nascimento (PHPN); informações maternas, antecedentes obstétricos e exames clínicos; e registros cotidianos inerentes às consultas.
Os dados de cadastro no PHPN verificados nos cartões foram: o local de realização do prénatal, a data da primeira consulta e a data da última menstruação; e as variáveis de registro diário foram relacionadas a um ou dois registros dos exames de tipagem (ABO) e fator(Rh) saguíneo, VDRL, urina (EAS), glicemia, hemoglobinemia, hematócrito, anti-HIV, HbsAg, toxoplasmose. Além disso, foram consideradas informações maternas, antecedentes pessoais e obstétricos, e exames clínicos. Nos campos direcionados aos registros cotidianos das consultas pré-natais avaliou-se a presença das datas das consultas, pesagem, medidas da pressão arterial, exame de toque, semanas de amenorreia, medidas da altura uterina, batimentos cardíacos fetais (BCF) e movimentos fetais (MF).

A classificação da modalidade dos serviços de saúde em: ESF, PACS e UBS tradicional foi obtida relacionando os nomes das unidades de saúde, presentes nos cartões ou os locais de residência das puérperas (quando não havia informação no cartão), com as informações públicas divulgadas pelas secretarias de saúde de cada município sobre bairros cobertos por tipos de serviços de saúde. As variáveis escolaridade e renda foram obtidas pelo relato das parturientes.

O banco de dados foi construído baseandose nas informações digitadas no software SPSS versão 12.0 (SPSS Inc., Chicago, Estados Unidos), submetido a exaustivo controle de qualidade e revisão. Os critérios utilizados para julgamento dos registros sobre os dados seguiram os parâmetros de Romero \& Cunha 15, que avaliam a completude dividindo-se o total de ausência de informação de uma variável pelo total da amostra. Em seguida, realizam a classificação segundo os níveis de qualidade: excelente (incompletude $<5 \%$ ), bom $(5 \% \leq$ incompletude $<10 \%)$, regular $(10 \% \leq$ incompletude $<20 \%)$, ruim $(20 \% \leq$ incompletude $<50 \%$ ), muito ruim (incompletude $\geq 50 \%$ ). Após a classificação, testes de qui-quadrado foram aplicados para testar a associação entre a modalidade do serviço de pré-natal e os registros das informações, adotando-se nível de significância estatística menor que $5 \%$.

\section{Resultados}

As condições socioeconômicas são diferentes nas áreas de cobertura dos serviços de saúde. Nas áreas cobertas pelas UBS a quantidade média de anos de estudos com aprovação das puérperas foi de 8,55 $\pm 2,8$, nas áreas de ESF 8,42 $\pm 2,6$ e nas de PACS $8,11 \pm 2,7$. Já a renda familiar mensal média foi menor nas áreas de cobertura do 
PACS (R\$ 981,24 $\pm 640,5)$ e maior nas de ESF (R\$ $1.145,92 \pm 709,196)$.

Na Região Metropolitana da Grande Vitória, quase metade das puérperas $(48,2 \%)$ possuía 7 ou mais consultas registradas. Na Figura 1, aponta-se o indicador sobre a quantidade de consultas pré-natais registradas nos cartões segundo a modalidade de serviços de saúde pública. Notase que nos cartões do ESF é mais expressiva a frequência de 7 ou mais consultas registradas e menos frequente o registro de 1 a 3 consultas prénatais, em relação ao PACS e à UBS.

A Tabela 1 apresenta os percentuais de ausência do registro das principais variáveis de cadastro e acompanhamento no PHPN. Observa-se que a data da primeira consulta é a que apresenta o menor percentual de campos em branco, sendo considerada de qualidade excelente. $\mathrm{O}$ registro sobre o local de realização do acompanhamento pré-natal diferiu significativamente entre os serviços de saúde, sendo menos registrados no PACS do que na UBS e no ESF.

Sobre o primeiro registro de realização de exames laboratoriais diagnósticos nos cartões, verifica-se que todos possuem nível de qualidade ruim $(20 \% \leq$ incompletude $<50 \%)$, exceto os exames de HbsAg e de toxoplasmose, que apresentam predominantemente nível muito ruim (incompletude $\geq 50 \%$ ). Contudo, constatase que nas UBS o percentual de incompletude é significativamente menor que na ESF e no PACS. Quando se avalia a repetição desses exames, todos, sem exceção, apresentam nível de registro muito ruim. Quanto à administração da vacina antitetânica, dose inicial ou de reforço, seu nível de registro foi muito ruim em todos os serviços de saúde. Porém, na ESF a ausência do registro foi menos acentuada que no PACS e na UBS.

A Tabela 2 mostra os percentuais de campos em branco sobre informações provenientes da anamnese e de alguns exames clínicos no acompanhamento pré-natal. Observa-se que as informações maternas possuem um nível de registro predominante muito ruim e ruim. Contudo, verifica-se que para o registro de todas as variáveis existe uma diferença entre modalidades de serviços, exceto para idade e tabagismo materno.

No que se refere aos antecedentes obstétricos, estes também são muito ruins, com exceção das variáveis abortos e partos. Mas, para registro das variáveis sobre a existência de algum recémnascido de baixo peso e nascimento do filho de maior peso, é no ESF que se conseguem os menores percentuais de campos sem informação. Já em relação aos exames clínicos, verifica-se que todos apresentam qualidade de registro muito

Figura 1

Percentuais de registros nos Cartões de Gestantes das consultas pré-natais, segundo a modalidade dos serviços de saúde pública. Região Metropolitana da Grande Vitória, Espírito Santo, Brasil, 2010.

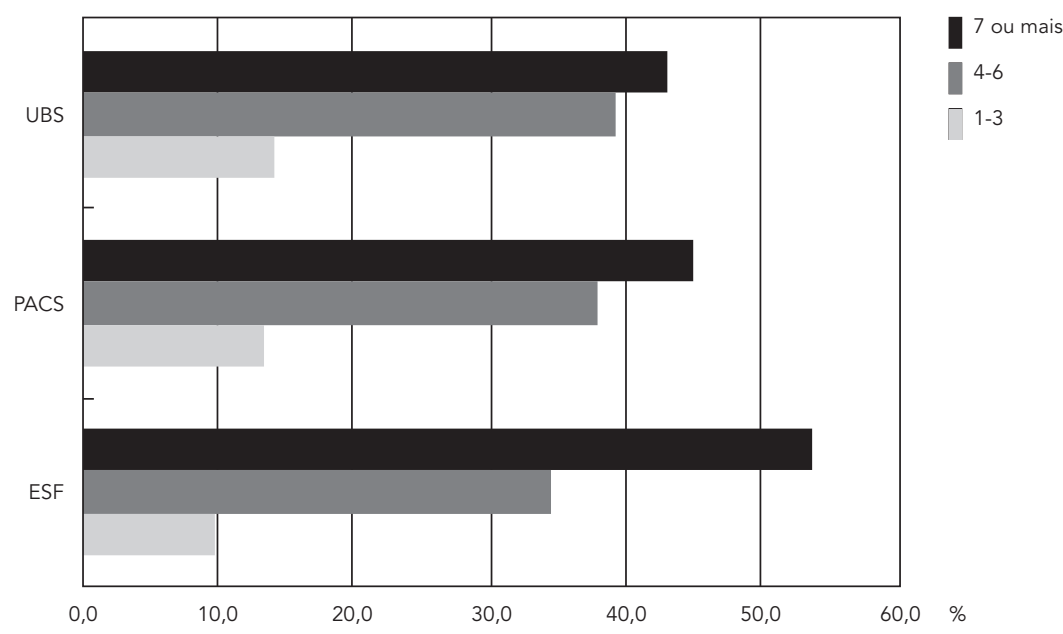

ESF: Estratégia Saúde da Família; PACS: Programa Agentes Comunitários de Saúde; UBS: unidades básicas de saúde. Nota: teste qui-quadrado $=9,64$; valor de $p=0,047$. 
Tabela 1

Avaliação da completude dos registros nos Cartões de Gestantes sobre variáveis do Programa de Humanização do Pré-natal e Nascimento (PHPN), segundo a modalidade de serviço de saúde pré-natal. Região Metropolitana da Grande Vitória, Espírito Santo, Brasil, 2010.

\begin{tabular}{|c|c|c|c|c|c|c|}
\hline \multirow[t]{2}{*}{ Registro } & \multirow{2}{*}{$\begin{array}{c}\text { Total } \% \\
(\mathrm{~N}=1.006)\end{array}$} & \multicolumn{3}{|c|}{ Serviço de saúde (\%) } & \multicolumn{2}{|c|}{ Estatística } \\
\hline & & $\begin{array}{c}\text { ESF } \\
(n=370)\end{array}$ & $\begin{array}{c}\text { PACS } \\
(n=143)\end{array}$ & $\begin{array}{c}\text { UBS } \\
(n=493)\end{array}$ & Qui-quadrado & Significância \\
\hline \multicolumn{7}{|l|}{ PHPN (cadastro) } \\
\hline Local do pré-natal & 46,7 & 41,9 & 62,9 & 45,6 & 21,2 & 0,001 \\
\hline Data da 1ạ consulta & 1,6 & 0,8 & 2,8 & 1,8 & 3,0 & 0,218 \\
\hline Data da última menstruação & 24,0 & 23,2 & 20,3 & 25,6 & 1,9 & 0,395 \\
\hline \multicolumn{7}{|l|}{ PHPN (registro diário) } \\
\hline Exame ABORh (1) & 37,2 & 38,4 & 35,0 & 36,9 & 0,3 & 0,848 \\
\hline Exame VDRL (1) & 28,2 & 28,4 & 28,0 & 28,2 & 0,0 & 0,999 \\
\hline Exame EAS (1) & 32,9 & 35,1 & 35,0 & 30,6 & 2,2 & 0,332 \\
\hline Glicemia (1) & 27,4 & 27,8 & 32,9 & 25,6 & 3,3 & 0,189 \\
\hline Hemoglobinemia (1) & 28,6 & 29,2 & 31,5 & 27,4 & 1,1 & 0,571 \\
\hline Hematócrito (1) & 34,8 & 35,9 & 39,9 & 32,5 & 3,3 & 0,195 \\
\hline Anti-HIV (1) & 34,0 & 37,0 & 30,8 & 32,7 & 2,2 & 0,341 \\
\hline HbsAg (1) & 50,0 & 52,2 & 43,4 & 50,3 & 2,6 & 0,267 \\
\hline Toxoplasmose (1) & 80,7 & 84,3 & 85,3 & 76,7 & 11,4 & 0,003 \\
\hline Vacina antitetânica & 59,3 & 56,2 & 68,5 & 59,0 & 8,3 & 0,016 \\
\hline Exame ABORh (2) & 90,5 & 90,3 & 90,9 & 90,5 & 1,1 & 0,574 \\
\hline Exame VDRL (2) & 65,5 & 64,6 & 72,7 & 64,1 & 5,2 & 0,074 \\
\hline Exame EAS (2) & 65,1 & 65,4 & 70,6 & 63,3 & 3,5 & 0,178 \\
\hline Glicemia (2) & 63,9 & 63,8 & 69,2 & 62,5 & 3,0 & 0,223 \\
\hline Hemoglobinemia (2) & 63,7 & 62,4 & 70,6 & 62,7 & 4,7 & 0,095 \\
\hline Hematócrito (2) & 67,9 & 65,7 & 76,2 & 67,1 & 7,5 & 0,024 \\
\hline Anti-HIV (2) & 74,8 & 76,8 & 74,1 & 73,4 & 0,9 & 0,646 \\
\hline $\operatorname{HbsAg}(2)$ & 80,3 & 79,5 & 83,9 & 79,9 & 2,9 & 0,238 \\
\hline Toxoplasmose (2) & 84,0 & 82,2 & 83,9 & 85,4 & 3,1 & 0,210 \\
\hline
\end{tabular}

Legenda: $\square$ Excelente $\square$ Ruim $\square$ Muito ruim.

ESF: Estratégia Saúde da Família; PACS: Programa Agentes Comunitários de Saúde; UBS: unidades básicas de saúde.

ruins. Contudo, no PACS e na UBS encontram-se significativamente mais campos em branco nos cartões para as variáveis: exames clínicos geral, de mamas, de pélvis e Papanicolau, do que na ESF.

Na Tabela 3, a qualidade dos registros foi avaliada considerando o total de campos em branco de cada variável dividido pelo total de consultas registradas, multiplicado por 100. Em seguida, julgou-se o nível de qualidade do registro das variáveis em cada Cartão de Gestante segundo os critérios de Romero \& Cunha 15.

Em mais de $80 \%$ dos cartões, as datas das consultas e as medidas da pressão arterial apresentaram um nível de preenchimento bom ou excelente. Entre $40 \%$ e $60 \%$ deles, constataram-se bons ou excelentes níveis para registro das semanas gestacionais e dos BCF. As pesagens nas consultas apresentaram altos percentuais de preenchimento. Porém, os cartões do ESF possuem um nível de completude significativamente maior que os do PACS e da UBS. Mais de $90 \%$ dos cartões indicaram nível de registro ruim ou muito ruim para o exame de toque vaginal. Entre $35 \%$ e $45 \%$ deles, tiveram níveis de completude ruins ou muito ruins para a medida da altura uterina e para a verificação dos MF, sem diferenças estatísticas entre os serviços de saúde.

\section{Discussão}

A avaliação dos Cartões das Gestantes permite inferir diretamente sobre a qualidade da assistência pré-natal nos serviços de saúde pública. Os registros revelam a passagem da gestante pelos serviços, os campos em branco nos cartões sugerem a ausência dessa passagem ou a pas- 
Avaliação da completude dos registros nos Cartões de Gestantes sobre variáveis maternas, obstétricas e clínicas, segundo a modalidade de serviço de saúde pré-natal. Região Metropolitana da Grande Vitória, Espírito Santo, Brasil, 2010.

\begin{tabular}{|c|c|c|c|c|c|c|}
\hline \multirow[t]{2}{*}{ Registro } & \multirow{2}{*}{$\begin{array}{c}\text { Total \% } \\
(\mathrm{N}=1.006)\end{array}$} & \multicolumn{3}{|c|}{ Serviço de saúde (\%) } & \multicolumn{2}{|c|}{ Estatísticas } \\
\hline & & $\begin{array}{c}\text { ESF } \\
(n=370)\end{array}$ & $\begin{array}{c}\text { PACS } \\
(n=143)\end{array}$ & $\begin{array}{c}\text { UBS } \\
(n=493)\end{array}$ & Qui-quadrado & Significância \\
\hline \multicolumn{7}{|l|}{ Informações maternas } \\
\hline Idade & 23,9 & 22,2 & 29,4 & 23,5 & 3,5 & 0,175 \\
\hline Alfabetização & 43,3 & 37,6 & 55,2 & 44,2 & 15,3 & 0,001 \\
\hline Anos de estudos & 45,3 & 39,2 & 57,3 & 46,5 & 16,2 & 0,001 \\
\hline Estado civil & 45,6 & 41,4 & 55,2 & 46,0 & 9,6 & 0,008 \\
\hline Antecedentes familiares & 57,7 & 52,4 & 65,0 & 59,4 & 9,9 & 0,007 \\
\hline Antecedentes pessoais & 76,9 & 71,6 & 79,0 & 80,3 & 12,7 & 0,002 \\
\hline Peso anterior à gestação & 49,9 & 35,9 & 75,5 & 52,9 & 74,4 & 0,001 \\
\hline Altura & 54,1 & 43,2 & 79,0 & 55,0 & 59,4 & 0,001 \\
\hline Tabagismo & 64,3 & 66,2 & 68,5 & 61,7 & 3,5 & 0,170 \\
\hline Data provável do parto & 28,6 & 24,9 & 36,4 & 29,2 & 6,8 & 0,033 \\
\hline \multicolumn{7}{|l|}{ Antecedentes obstétricos } \\
\hline Número de abortos & 46,0 & 47,6 & 44,1 & 45,4 & 0,4 & 0,827 \\
\hline Número de partos & 27,2 & 27,8 & 26,6 & 27,0 & 0,0 & 0,976 \\
\hline Número de partos vaginais & 49,4 & 53,0 & 50,3 & 46,5 & 3,4 & 0,184 \\
\hline Número de partos cesáreos & 72,9 & 73,8 & 73,4 & 72,0 & 0,4 & 0,832 \\
\hline Número de filhos nascidos vivos & 53,2 & 53,8 & 55,2 & 52,1 & 0,7 & 0,718 \\
\hline Número de nascidos mortos & 90,0 & 90,8 & 89,5 & 89,5 & 0,2 & 0,885 \\
\hline Número de filhos que vivem & 60,2 & 60,8 & 65,0 & 58,4 & 2,6 & 0,267 \\
\hline Número de filhos mortos ( $1^{\text {a }}$ semana) & 94,8 & 96,5 & 94,4 & 93,7 & 4,0 & 0,134 \\
\hline Número de filhos mortos (após $1^{\text {a }}$ semana) & 94,9 & 96,5 & 93,0 & 94,3 & 2,0 & 0,368 \\
\hline Recém-nascido (peso < 2.500g) & 78,6 & 75,7 & 83,9 & 79,3 & 7,2 & 0,027 \\
\hline Recém-nascido (de maior peso) & 77,0 & 73,8 & 83,9 & 77,5 & 9,2 & 0,010 \\
\hline \multicolumn{7}{|l|}{ Exames clínicos } \\
\hline Exame clínico geral & 85,3 & 83,0 & 91,6 & 85,2 & 10,9 & 0,004 \\
\hline Mamas & 86,8 & 84,3 & 91,6 & 87,2 & 9,7 & 0,008 \\
\hline Odontológico & 94,0 & 93,5 & 95,1 & 94,1 & 4,6 & 0,101 \\
\hline Pélvis & 92,2 & 90,0 & 93,7 & 93,5 & 11,3 & 0,004 \\
\hline Papanicolaou & 91,1 & 87,8 & 93,7 & 92,7 & 16,4 & 0,001 \\
\hline Colposcopia & 94,9 & 95,1 & 95,1 & 94,7 & 1,9 & 0,391 \\
\hline Cérvix & 94,1 & 93,2 & 94,4 & 94,7 & 5,4 & 0,069 \\
\hline
\end{tabular}

Legenda: $\square$ Ruim $\square$ Muito ruim.

ESF: Estratégia Saúde da Família; PACS: Programa Agentes Comunitários de Saúde; UBS: unidades básicas de saúde.

sagem nos serviços sem registro (sub-registro). As anotações das consultas revelam o acesso à assistência pré-natal, entendido como a entrada dos usuários nos serviços de saúde e a continuidade do cuidado 16 .

Todavia, deve-se considerar que o acesso e seus matizes incorporam as dimensões relacionadas à disponibilidade dos serviços em atender às necessidades em saúde, aos meios de mobilização de recursos financeiros para obter cuidados e à aceitabilidade envolvida na relação entre serviços de saúde e usuários ${ }^{17}$. Além disso, percebe-se que acesso, integralidade e equidade nos serviços de saúde são interdependentes e essenciais à qualidade do cuidado 18 .

No que se refere ao processo de assistência pré-natal, que possui um arcabouço tecnológico bem definido, não se pode pensar que apenas a passagem burocrática pelos serviços de saúde promove qualidade da atenção, visto que a resolutividade relaciona-se à capacidade de um serviço cumprir o que lhe compete. Contudo, a 
Avaliação da completude dos registros nos Cartões de Gestantes sobre variáveis de registro cotidiano nas consultas pré-natais, segundo a modalidade de serviço de saúde. Região Metropolitana da Grande Vitória, Espírito Santo, Brasil, 2010.

\begin{tabular}{|c|c|c|c|c|c|}
\hline \multirow[t]{2}{*}{ Registro/Qualidade } & \multicolumn{3}{|c|}{ Serviço de saúde (\%) } & \multicolumn{2}{|c|}{ Estatísticas } \\
\hline & $\begin{array}{c}\text { ESF } \\
(n=370)\end{array}$ & $\begin{array}{c}\text { PACS } \\
(n=143)\end{array}$ & $\begin{array}{c}\text { UBS } \\
(n=493)\end{array}$ & Qui-quadrado & Significância \\
\hline \multicolumn{6}{|l|}{ Datas das consultas } \\
\hline Muito ruim/Ruim & 4,6 & 2,1 & 3,7 & 3,7 & 0,442 \\
\hline Regular & 3,5 & 5,6 & 5,5 & & \\
\hline Bom/Excelente & 90,0 & 88,1 & 87,8 & & \\
\hline \multicolumn{6}{|l|}{ Pesagem nas consultas } \\
\hline Muito ruim/Ruim & 8,1 & 9,1 & 14,8 & 10,1 & 0,039 \\
\hline Regular & 9,5 & 9,8 & 8,9 & & \\
\hline Bom/Excelente & 78,6 & 76,9 & 73,0 & & \\
\hline \multicolumn{6}{|c|}{ Medidas da pressão arterial } \\
\hline Muito ruim/Ruim & 8,1 & 6,3 & 11,4 & 4,9 & 0,298 \\
\hline Regular & 7,3 & 8,4 & 7,1 & & \\
\hline Bom/Excelente & 82,2 & 80,4 & 77,5 & & \\
\hline \multicolumn{6}{|c|}{ Exame de toque nas consultas } \\
\hline Muito ruim/Ruim & 96,8 & 95,8 & 95,7 & 5,6 & 0,234 \\
\hline Regular & 0,8 & 0,0 & 0,2 & & \\
\hline Bom/Excelente & 0,3 & 0,0 & 1,0 & & \\
\hline \multicolumn{6}{|c|}{ Cálculos das semanas gestacionais } \\
\hline Muito ruim/Ruim & 29,7 & 35,7 & 34,5 & 6,8 & 0,149 \\
\hline Regular & 13,5 & 11,2 & 16,0 & & \\
\hline Bom/Excelente & 54,6 & 49,0 & 46,5 & & \\
\hline \multicolumn{6}{|c|}{ Medidas da altura uterina } \\
\hline Muito ruim/Ruim & 44,6 & 43,4 & 38,9 & 8,8 & 0,065 \\
\hline Regular & 22,4 & 14,0 & 20,1 & & \\
\hline Bom/Excelente & 30,8 & 38,5 & 37,9 & & \\
\hline \multicolumn{6}{|c|}{ Verificação dos batimentos cardíacos fetais } \\
\hline Muito ruim/Ruim & 33,2 & 34,3 & 32,9 & 9,4 & 0,052 \\
\hline Regular & 21,1 & 11,9 & 14,6 & & \\
\hline Bom/Excelente & 43,5 & 49,7 & 49,5 & & \\
\hline \multicolumn{6}{|c|}{ Verificação dos movimentos fetais } \\
\hline Muito ruim/Ruim & 47,8 & 39,9 & 44,6 & 9,2 & 0,057 \\
\hline Regular & 15,1 & 17,5 & 10,8 & & \\
\hline Bom/Excelente & 34,9 & 38,5 & 41,6 & & \\
\hline
\end{tabular}

ESF: Estratégia Saúde da Família; PACS: Programa Agentes Comunitários de Saúde; UBS: unidades básicas de saúde.

insuficiência e despreparo dos serviços de saúde podem levar ao comprometimento da atenção quando as tecnologias leves, leve-duras e duras existem mas não são empregadas de modo adequado 19 .

No entanto, a avaliação sobre o preenchimento dos cartões de pré-natal pode desvendar apenas uma parte da "cesta de necessidades" das gestantes e se elas estão sendo atendidas em alguns aspectos preconizados pelo PHPN, que se traduzem em quantidades de consultas, exames clínicos, físicos e laboratoriais, além da administração medicamentosa. Neste estudo, os resultados a respeito da assistência pré-natal explicam em parte os indicadores de mortalidade materna e infantil na Região Metropolitana da Grande Vitória.

Menos da metade dos cartões apresentou sete ou mais consultas registradas. Percentuais em torno de $70 \%$ foram descritos por Coutinho et al. 20 em Juiz de fora, Minas Gerais, e valores em torno de $30 \%$ foram evidenciados por Lima et al. 21 
em Salvador, Bahia. Isso mostra que o acesso à assistência pré-natal na Região Metropolitana da Grande Vitória encontra-se deficitário. Em relação às variáveis de cadastro e acompanhamento indicadas nos protocolos do PHPN, os cartões provenientes de áreas com cobertura da ESF também apresentaram melhores resultados; já os cartões do PACS apresentaram sistematicamente os piores níveis de completude.

Deve-se destacar que a realização de exames depende de um fluxo que se estabelece a partir do momento em que a gestante realiza o encontro com o pré-natalista. Isso requer a solicitação do exame, a procura pelo laboratório, a realização do exame, a emissão do resultado, o retorno ao profissional, e, por fim, a anotação no cartão da gestante. Supõe-se que os piores resultados do PACS possam ser explicados pelo fato desses serviços serem ofertados em áreas geográficas de maior índice de pobreza, áreas distantes dos centros urbanos. Por exemplo, no Município de Cariacica (PIB $=\mathrm{R} \$ 9.806$ ) o PACS cobriu cerca de $45 \%$ das puérperas, ao passo que aproximadamente $30 \%$ das puérperas residentes no $\mathrm{Mu}$ nicípio de Guarapari, situado a $51 \mathrm{~km}$ da capital, também foram cobertas pelo PACS no pré-natal (Instituto de Apoio à Pesquisa e ao Desenvolvimento Jones dos Santos Neves. http://www.ijsn. es.gov.br/, acessado em 09/Jul/2010).

Esses profissionais podem ter dificuldade no fluxo de envio e recebimento dos exames laboratoriais, bem como no encaminhamento de gestantes de alto risco para a realização de exames específicos adequados à atenção pré-natal, o que identifica um grave problema de acesso. Por outro lado, nessas áreas as gestantes podem não aderir ao pré-natal devido ao baixo nível de escolaridade e de renda. Além disso, deve-se ressaltar que por ser considerado um modelo de assistência de transição entre a UBS e o ESF, o PACS está sendo gradativamente extinto, o que também pode contribuir para o seu pior desempenho.

Para os exames que possuem obrigatoriedade quanto à realização, no mínimo uma vez, segundo o PHPN, os níveis de registro nos cartões foram ruins, excetuando-se a realização do segundo exame de hematócrito que foi significativamente maior na ESF. Quando se avalia a repetição obrigatória dos exames de VDRL, EAS e glicemia, os níveis de registro são piores ainda, com mais de $50 \%$ de incompletude. Resultados similares foram encontrados por Vettore et al. 22 no Município do Rio de Janeiro: nível de registro regular para o exame de $\mathrm{Hb}$; ruim para o VDRL; e muito ruins para a testagem anti-HIV e EAS. Carvalho \& Araújo 23 avaliaram cartões e prontuários de assistência às grávidas de alto risco na cidade de Recife, Pernambuco, e encontraram um nível de registro regular do fator Rh; ruim dos exames de VDRL, EAS, Hb e glicemia; e nível Muito ruim da testagem anti-HIV. Ressalte-se a gravidade desses resultados, contrastando com o presente estudo, composto principalmente de gestantes de baixo risco.

A abordagem da sífilis no pré-natal é considerada um dos marcadores da qualidade da assistência ${ }^{24}$. Embora o nível de completude para repetição do exame VDRL tenha sido considerado muito ruim, aproximadamente 35\%, está próximo dos descritos por Campos et al. 25 e por Coutinho et al. 26, e muito melhor do que o descrito por Lima et al. 21 , que foi cerca de $10 \%$. Isso sugere que, apesar de distante do ideal, a pesquisa da sífilis está sendo valorizada na Região Metropolitana da Grande Vitória de modo similar a outros municípios das regiões Sul e Sudeste do Brasil.

Outro exame essencial é a testagem anti-HIV, importante para a descoberta da doença e prevenção da transmissão vertical. Nesta avaliação, a ausência de realização desse exame chegou a 35\%, sem diferenças entre os serviços saúde, muito semelhante ao encontrado numa cidade do interior de Minas Gerais 20. Morimura et al. 27 destacaram que cerca de $40 \%$ das mulheres chegam ao momento do parto sem o resultado da testagem. Problemas de diversas naturezas podem explicar esses resultados, mas sugere-se que o conjunto de problemas esteja mais relacionado à organização do SUS 28 .

Quanto ao registro do exame sobre pesquisa de toxoplasmose, as UBS apresentaram resultado significativamente melhor do que as demais modalidades de serviços. Sua realização não é obrigatória no PHPN 29, mas é recomendada sempre que possível 30 . O rastreamento da toxoplasmose no pré-natal é indicado pelos ginecologistas obstetras devido às graves repercussões fetais 31 . $\mathrm{O}$ que pode explicar as diferenças de registros entre as modalidades de serviços de acompanhamento pré-natal é a presença de especialistas, geralmente médicos ginecologistas, envolvidos na condução do pré-natal nas UBS. Ao passo que no PACS e na ESF são os enfermeiros e médicos generalistas quem acompanham as gestantes e solicitam os exames 32 .

Quanto à administração obrigatória da vacina antitetânica 29 , importante para a prevenção do tétano neonatal 33, apresentou maior quantidade de registros nos cartões da ESF. Isso é corroborado por Guimarães et al. 34 que revelam expressivo aumento da cobertura vacinal nas áreas da implantação da ESF. Porém, o registro dessa informação foi muito inferior ao encontrado no estudo de Trevisan et al. 35, mais de 90\%, que considerou a informações do cartão, do prontuário e da própria puérpera, possivelmente porque as 
fontes de informação foram somadas para a obtenção dos resultados, o que não é o caso deste trabalho que considerou apenas cartões.

Os registros das informações maternas e antecedentes obstétricos dependem de uma anamnese bem conduzida. Ao avaliar o preenchimento desses quesitos, o PACS apresentou os piores resultados, exceto para o registro dos antecedentes pessoais, enquanto nos cartões do ESF foram menores os percentuais de ausência de informação. Em síntese, nota-se que essas informações são muito desvalorizadas na assistência pré-natal.

A ausência desses dados nos cartões não pode ser totalmente explicada pelo uso do cartão como fonte de informação, pois Oba \& Tavares 36 também observaram ausência de informações maternas em todos os prontuários de acompanhamento pré-natal, além de percentual expressivo de prontuários sem registro dos antecedentes obstétricos. No presente estudo, verificou-se que essas variáveis tiveram seus registros muito negligenciados nos cartões, sugerindo o desprezo dos profissionais da saúde por eles em todas as modalidades de serviços públicos. Essa deficiência nos registros também foi encontrada em todos os Cartões de Gestantes do ESF avaliados no estudo de Costa et al. 37 . Diferentemente, o PACS e a UBS apresentaram resultados piores que a ESF.

Este trabalho revelou que os registros dos exames clínicos foram negligenciados no cartão da gestante por mais de $90 \%$ dos profissionais que conduziram o pré-natal. Esse fenômeno também foi descrito em unidades de referência à gravidez de alto risco, onde os exames de mamas e ginecológicos não constavam em $80 \%$ dos prontuários nem nos cartões de pré-natal 23. De modo semelhante, percentuais próximos a esse foram encontrados no SUS do Município de Rio Grande, Rio Grande do Sul 38.

Deve-se considerar que os resultados foram semelhantes entre acompanhamento pré-natal de profissionais médicos e enfermeiros, visto que o PACS e a UBS apresentaram resultados parecidos. Estudos que avaliaram a assistência prénatal praticada por enfermeiros e médicos, em diferentes estados brasileiros, revelaram que exames de mamas e ginecológicos são realizados por cerca de $40 \%$ a $60 \%$ destes profissionais 35,36,39,40. Quanto ao registro das atividades clínicas de rotina realizadas nas consultas de acompanhamento pré-natal e que devem ser repetidas em cada visita, nota-se um padrão diferente: são mais elevados os níveis de completude do que para os itens analisados previamente. Entretanto, o PACS permanece com o pior desempenho, a UBS com níveis intermediários e o ESF com os melhores indicadores.
As datas das consultas, pesagem, medidas da pressão arterial, cálculos das semanas gestacionais e verificação dos BCF tiveram bons ou excelentes níveis de registro. Resultados semelhantes foram observados por Coutinho et al. para Juiz de Fora em 200220 e 2006 26. Já o estudo de Vettore et al. 22 encontrou resultados mais expressivos ainda, chegando ao registro de $95 \%$ da pesagem e da medida da pressão arterial. A comparação com esses trabalhos revelam um déficit na qualidade da assistência pré-natal no SUS da Região Metropolitana da Grande Vitória. Neste estudo foram ruins e muito ruins os registros do exame de toque, medida da altura uterina e verificação dos MF, em concordância com o trabalho em um município do Sudeste brasileiro 20,26 que também referiu níveis de preenchimento nos cartões destas variáveis bastante reduzidos.

Nota-se que as atividades atribuídas aos profissionais técnicos ou auxiliares de enfermagem como, por exemplo, pesagem e verificação da pressão arterial, são melhores registradas do que aquelas realizadas por médicos e enfermeiros. Isso sugere que os profissionais de nível técnico são formados para cumprir os protocolos de assistência estabelecidos 41 .

Como visto, de um modo geral, os registros foram significativamente mais valorizados nos cartões provenientes de puérperas cobertas pelo ESF. Esse fenômeno deve ser relacionado ao nível de organização diferenciado do ESF, onde atuam diferentes categorias profissionais no processo de trabalho e consequentemente no preenchimento do cartão do pré-natal ${ }^{32}$. Parece ser coerente afirmar que a qualidade do preenchimento dos cartões de gestantes tem maior possibilidade de acontecer no ESF do que no PACS e UBS. Como mostra a análise de discurso de Melo \& Coelho 42 sobre assistência pré-natal de adolescentes nas USF, o preenchimento do Cartão da Gestante por ACS, enfermeiros e médicos é parte constituinte da assistência.

Entretanto, para algumas dimensões não se encontrou diferenças estatisticamente significantes entre cartões de gestantes provenientes de áreas cobertas pelo ESF, PACS e UBS. Isso evidencia que os serviços de assistência pré-natal estão num momento de transição, longe dos requisitos esperados, segundo os critérios das políticas de saúde nacionais. Mesmo com toda a filosofia do ESF, centrada no trabalho em equipe, na interdisciplinaridade e nos princípios de solidariedade, percebe-se que a mesma não conseguiu superar limitações de uma prática fragmentada.

As marcantes diferenças entre o registro das atividades desempenhadas por profissionais de nível superior e de nível técnico traduzem a ausência de interdisciplinaridade. Isso se destaca 
como um dos impedimentos para o cuidado humanizado no pré-natal, principalmente quando a lógica do trabalho é centrada na produtividade, com consultas rápidas e sem profundidade 43 . Embora sejam essenciais a uma gravidez segura, deve-se destacar que a proposta do ESF para o processo de assistência pré-natal vai além da realização de exames físicos, clínicos e laboratoriais. A formação de vínculos e correponsabilização entre profissionais e usuárias também são aspectos cruciais na atenção.

Deve-se considerar que os resultados da assistência pré-natal, apresentados neste estudo, levaram em conta critérios bastante objetivos de necessidade em saúde, entendida como a necessidade de consumir tecnologias para melhorar as condições de vida e saúde de mulheres e crianças 19 , visto que foram avaliados os registros nos cartões das tecnologias que deveriam ser utilizadas no acompanhamento gestacional. Também existem limitações quanto à fonte de informação, pois se considerou apenas o registro nos Cartões de Gestantes. Existe a possibilidade de procedimentos serem realizados e não registrados ou de serem registrados e não realizados. Além disso, há limitações inerentes ao desenho de estudo, que não é capaz de testar hipóteses sobre vínculos causais, mas apenas sugerir associações no local e no tempo específico.

Deve-se reconhecer também que no nível micropolítico do trabalho em saúde, em que o processo de assistência pré-natal acontece, existe a articulação de conhecimentos dos profissionais de saúde com a política, gestão e planejamento, inerentes ao nível macropolítico ${ }^{18}$. Nesse sentido, a construção da integralidade para gestantes no SUS deverá tentar incorporar ao processo de trabalho, além do que já tem sido preconizado pelas políticas de saúde materno-infantil, a possibilidade de identificar e solucionar necessidades em saúde, por meio da reorganização dos serviços de atenção básica em saúde ${ }^{44}$.

\section{Conclusão}

Embora a ausência de registro não signifique exatamente a ausência de realização de procedimentos pré-natais, de um modo geral os serviços de assistência pré-natal na Região Metropolitana da Grande Vitória, avaliados por meio dos Cartões de Gestantes, foram ruins ou muito ruins. Contudo, a ESF apresentou níveis de qualidade um pouco melhor, enquanto o PACS demonstrou sistematicamente os piores resultados.

Dentre os itens avaliados, o registro de informações advindas da anamnese pré-natal foi o menos preenchido nos Cartões das Gestantes, principalmente informações maternas e antecedentes obstétricos. Além disso, os exames clínicos de mama e toque vaginal, que dependem de maior proximidade entre gestantes e profissionais pré-natalistas, não foram realizados na maioria dos acompanhamentos pré-natais. Isso reflete a micropolítica do trabalho na assistência pré-natal.

A questão crucial, relacionada aos exames laboratoriais, sejam estes obrigatórios ou apenas recomendados pela política brasileira de saúde materno-infantil, merece atenção porque é dependente dos fluxos de referência e contrarreferência que qualificam o acesso e a integralidade no SUS. Nesse caso, os resultados desfavoráveis indicam que no nível macropolítico estratégias devem ser desenvolvidas para a melhoria do acesso e construção da integralidade na atenção básica da Região Metropolitana da Grande Vitória.

Questiona-se se os profissionais de saúde estão preparados para executar adequadamente o processo de trabalho da assistência pré-natal nos serviços de saúde pública. Portanto, investigações científicas sobre a gestão do trabalho na assistência pré-natal no SUS parecem sinalizar um caminho que aponte soluções para a melhoria da qualidade do cuidado à gestante. 


\section{Resumo}

Objetivou-se avaliar a completude de informações sobre assistência pré-natal nos cartões de gestantes, segundo a cobertura do serviço de saúde pública: Estratégia Saúde da Família (ESF), Programa Agentes Comunitários de Saúde (PACS) e unidade básica de saúde (UBS) tradicional, na Região Metropolitana da Grande Vitória, Espírito Santo, Brasil. Um estudo seccional selecionou aleatoriamente 1.006 cartões de puérperas em maternidades da região escolhida para a pesquisa. A completude dos cartões de pré-natal foi avaliada pelos critérios de Romero \& Cunha, que mensuram a qualidade numa escala decrescente de excelente (incompletude $<5 \%$ ) a muito ruim (incompletude > 50\%). De forma geral, o preenchimento das informações nos cartões foi ruim (incompletude $>$ 20\%), contudo, no ESF o cartão é mais bem preenchido do que no PACS e nas UBS, principalmente para vacinação antitetânica $(p=0,016)$ e pesagem gestacional $(p=0,039)$. Concluiu-se que a qualidade da assistência pré-natal no SUS da Região Metropolitana da Grande Vitória encontra-se distante das políticas nacionais de saúde materno-infantil.

Saúde Materno-Infantil; Cuidado Pré-Natal; Avaliação em Saúde; Qualidade da Assistência à Saúde; Políticas Públicas de Saúde

\section{Referências}

1. Instituto Nacional de Assistência Médica da Previdência Social. Assistência pré-natal. Brasília: Divisão Nacional de Saúde Materno-Infantil, Secretaria Nacional de Programas Especiais de Saúde, Ministério da Saúde; 1988.

2. Perim EB, Passos ADC. Hepatite B em gestantes atendidas pelo Programa do Pré-natal da Secretaria Municipal de Saúde de Ribeirão Preto, Brasil: prevalência da infecção e cuidados prestados aos recém-nascidos. Rev Bras Epidemiol 2005; 8: 272-81.

3. Essén B, Laurell L, Peña R, Ostergren PO, Liljestrand J. Antenatal cards - what should they contain? J Trop Pediatr 1994; 40:130-2.

4. Área Técnica Saúde da Mulher, Secretaria de Políticas de Saúde, Ministério da Saúde. Programa de Humanização no Pré-natal e Nascimento. Rev Bras Saúde Matern Infant 2002; 2:69-71.

\section{Colaboradores}

E. T. Santos Neto participou da concepção do estudo, planejamento da pesquisa, coleta de dados, redação do artigo, análise estatística e revisão final. A. E. Oliveira participou da concepção do estudo, planejamento da pesquisa, redação do artigo e revisão final. E. Zandonade participou da concepção do estudo, planejamento da pesquisa, redação do artigo, análise estatística e revisão final. S. G. N. Gama participou do planejamento da pesquisa, redação do artigo e revisão final. M. C. Leal participou do planejamento da pesquisa, redação do artigo, análise estatística e revisão final.

\section{Agradecimentos}

À Fundação de Amparo à Pesquisa do Espírito Santo (FAPES).
5. Mishima FC, Scochi CGS, Ferro MAR, Lima RAG, Costa IAR. Declaração de nascido vivo: análise do seu preenchimento no Município de Ribeirão Preto, São Paulo, Brasil. Cad Saúde Pública 1999; 15:387-95.

6. Andreucci CB, Cecatti JG. Desempenho de indicadores de processo do Programa de Humanização do Pré-natal e Nascimento no Brasil: uma revisão sistemática. Cad Saúde Pública 2011; 27:1053-64.

7. Dode MASO, Santos IS. Validade do auto-relato de diabete mellitus gestacional no pós-parto imediato. Cad Saúde Pública 2009; 25:251-8.

8. Alonso-Silva MSS. Análise da assistência pré-natal na rede pública do Município do Rio de Janeiro, 2007-2008 [Dissertação de Mestrado]. Rio de Janeiro: Escola Nacional de Saúde Publica Sergio Arouca, Fundação Oswaldo Cruz; 2009. 
9. Draper J, Field S, Thomas H, Hare MJ. Should women carry their antenatal records. BMJ 1986; 292:603.

10. Branco MAF. Informação em saúde como elemento estratégico para a gestão. In: Ministério da Saúde, organizador. Gestão municipal de saúde: textos básicos. Brasília: Ministério da Saúde; 2001. p. 163-9.

11. Paim JS. Modelos de atenção e vigilância da saúde. In: Rouquayrol MZ, Almeida Filho, N, organizadores. Epidemiologia e saúde. 6a Ed. Rio de Janeiro: Editora Medsi; 2003. p. 567-86.

12. Secretaria Executiva, Ministério da Saúde. Programa Agentes Comunitários de Saúde. Brasília: Ministério da Saúde; 2001.

13. Secretaria de Políticas de Saúde, Ministério da Saúde. Saúde da família: uma estratégia para a reorientação do modelo assistencial. Brasília: Ministério da Saúde; 1997.

14. Piccini RX, Facchini LA, Tomasi E, Thumé E, Silveira DS, Siqueira FV, et al. Efetividade da atenção pré-natal e de puericultura em unidades básicas de saúde do Sul e do Nordeste do Brasil. Rev Bras Saúde Matern Infant 2007; 7:75-82.

15. Romero DE, Cunha CB. Avaliação da qualidade das variáveis sócio-econômicas e demográficas dos óbitos de crianças menores de um ano registrados no Sistema de Informações sobre Mortalidade do Brasil (1996/2001). Cad Saúde Pública 2006; 22:673-81.

16. Andersen RM. Revisiting the behavioral model and access to medical care: does it matter? J Health Soc Behav 1995; 36:1-10.

17. Thiede M, Akweongo P, McIntyre D. Exploring the dimensions of access. In: McIntyre D, Mooney G, editors. The economics of health equity. Cambridge: Cambridge University Press; 2007. p. 103-23.

18. Pinheiro R, Mattos RA. Sentidos da integralidade na atenção e no cuidado à saúde. Rio de Janeiro: Instituto de Medicina Social, Universidade do Estado do Rio de Janeiro/ABRASCO; 2006.

19. Merhy EE. Saúde: a cartografia do trabalho vivo. São Paulo: Editora Hucitec; 2002.

20. Coutinho T, Teixeira MTB, Dain S, Sayd JD, Coutinho LM. Adequação do processo de assistência pré-natal entre as usuárias do Sistema Único de Saúde em Juiz de Fora - MG. Rev Bras Ginecol Obstet $2003 ; 25: 717-24$.

21. Lima BGC, Costa MCN, Dourado MIC. Avaliação da qualidade do rastreamento de HIV/AIDS e sífilis na assistência pré-natal. Epidemiol Serv Saúde 2008; 17:123-53.

22. Vettore MV, Dias M, Domingues RMSM, Vettore MV, Leal MC. Cuidados pré-natais e avaliação do manejo da hipertensão arterial em gestantes do SUS no Município do Rio de Janeiro, Brasil. Cad Saúde Pública 2011; 27:1021-34.

23. Carvalho VCP, Araújo TVB. Adequação da assistência pré-natal em gestantes atendidas em dois hospitais de referência para gravidez de alto risco do Sistema Único de Saúde, na Cidade de Recife, Estado de Pernambuco. Rev Bras Saúde Matern Infant 2007; 7:309-17.
24. Saraceni V, Guimarães MHFS, Theme Filha MM, Leal MC. Mortalidade perinatal por sífilis congênita: indicador da qualidade da atenção à mulher e à criança. Cad Saúde Pública 2005; 21:1244-50.

25. Campos ALA, Araújo MAL, Melo SP, Gonçalves MLC. Epidemiologia da sífilis gestacional em Fortaleza, Ceará, Brasil: um agravo sem controle. Cad Saúde Pública 2010; 26:1747-55.

26. Coutinho T, Monteiro MFG, Sayd JD, Teixeira MTB, Coutinho CM, Coutinho LM. Monitoramento do processo de assistência pré-natal entre as usuárias do Sistema Único de Saúde em município do Sudeste brasileiro. Rev Bras Ginecol Obstet 2010; 32:563-9.

27. Morimura MCR, Mendes MDC, Souza AI, Alencar LCA. Frequência de testagem rápida para o HIV durante a admissão para o parto em puérperas no Instituto Materno Infantil Prof. Fernando Figueira, IMIP. Rev Bras Saúde Matern Infant 2011; 6 Suppl 1:S69-76.

28. Almeida CAL, Tanaka OY. Perspectiva das mulheres na avaliação do Programa de Humanização do Pré-natal e Nascimento. Rev Saúde Pública 2009; 43:98-104.

29. Brasil. Portaria n. 569 de 1 de junho de 2000. http:// www.saude.mg.gov.br/atos_normativos/legisla caosanitaria/estabelecimentos-de-saude/atendi mento-humanizado/Portaria_569.pdf (acessado em 09/Jul/2007).

30. Área Técnica de Saúde da Mulher, Departamento de Ações Programáticas Estratégicas, Secretaria de Atenção à Saúde, Ministério da Saúde. Pré-natal e puerpério: atenção qualificada e humanizada - manual técnico. Brasília: Ministério da Saúde; 2006.

31. Amorim MMR, Melo ASO. Avaliação dos exames de rotina no pré-natal (Parte 1). Rev Bras Ginecol Obstet 2009; 31:148-55.

32. Coradini SR, Azzi LMW, Mendonça CS, Oliveira DL. Processo de trabalho no Programa Saúde da Família em Porto Alegre: a discussão das competências profissionais como proposta de mudança. Boletim da Saúde 2004; 18:169-74.

33. Vieira LJ, Oliveira MHP, Lefèvre F. O uso da expressão - mal-de-sete-dias - por mães de crianças que morreram de tétano neonatal em Minas Gerais (1997-2002). Texto \& Contexto Enferm 2006; 15:51-9.

34. Guimarães TMR, Alves JGB, Tavares MMF. Impacto das ações de imunização pelo Programa Saúde da Família na mortalidade infantil por doenças evitáveis em Olinda, Pernambuco, Brasil. Cad Saúde Pública 2009; 25:868-76.

35. Trevisan MR, De Lorenzi DRS, Araujo NM, Esber K. Perfil da assistência pré-natal entre usuárias do Sistema Único de Saúde em Caxias do Sul. Rev Bras Ginecol Obstet 2002; 24:293-9.

36. Oba MDV, Tavares MSG. A precariedade dos registros de assistência pré-natal em uma Unidade Básica de Saúde no Município de Ribeirão Preto - SP. Rev Latinoam Enferm 1998; 6:53-61. 
37. Costa GD, Cotta RMM, Reis JR, Siqueira-Batista R, Gomes AP, Franceschini SCC. Avaliação do cuidado à saúde da gestante no contexto do Programa Saúde da Família. Ciênc Saúde Coletiva 2009; 14 Suppl 1:1347-57.

38. Gonçalves CV, Costa JSD, Duarte G, Marcolin AC, Lima LCV, Garlet G, et al. Avaliação da frequência de realização do exame físico das mamas, da colpocitologia cervical e da ultrassonografia obstétrica durante a assistência pré-natal: uma inversão de valores. Rev Assoc Med Bras 2009; 55:290-5.

39. Cunha MA, Mamede MV, Dotto LMG, Mamede FV. Assistência pré-natal: competências essenciais desempenhadas por enfermeiros. Esc Anna Nery Rev Enferm 2009; 13:145-53.

40. Narchi NZ. Atenção pré-natal por enfermeiros na Zona Leste da Cidade de São Paulo - Brasil. Rev Esc Enferm USP 2010; 44:266-73.
41. Conselho Nacional de Educação, Ministério da Educação. Resolução no 4 de 22 dezembro de 1999. Dispõe sobre diretrizes curriculares nacionais para a educação profissional de nível técnico. Diário Oficial da União 1999; 22 dez.

42. Melo MCP, Coelho EAC. Integralidade e cuidado a grávidas adolescentes na Atenção Básica. Ciênc Saúde Coletiva 2011; 16:2549-58.

43. Zampieri MFM, Erdmann AL. Cuidado humanizado no pré-natal: um olhar para além das divergências e convergências. Rev Bras Saúde Matern Infant 2010; 10:359-67.

44. Pinheiro R, Mattos RA. Construção da integralidade: cotidiano, saberes e práticas em saúde. $4^{\text {a }}$ Ed. Rio de Janeiro: Instituto de Medicina Social, Universidade do Estado do Rio de Janeiro/ABRASCO; 2007.

Recebido em 27/Fev/2012

Versão final reapresentada em 16/Mai/2012

Aprovado em 28/Mai/2012 